10.21611/qirt.2016.b

\title{
QIRT 2016
}

Keynote Talk

\author{
Approaches to Thermographic Detection, Measurement and Characterization \\ Dr. Steven M. Shepard \\ Thermal Wave Imaging, Inc.
}

\begin{abstract}
Although there are numerous historical examples of thermal methods for NDT (e.g. thermally sensitive paints and tapes), the modern era of image-based Thermographic NDT (TNDT) began in the late 1960's, with the advent of the IR video camera. In the ensuing 50 years, the discipline has evolved from a laboratory curiosity to a widely implemented NDT method in the aerospace, automotive and power generation industries. Progress in the field must be attributed, in part, to enormous advances in IR camera and computer technology. The commercial release of focal plane array camera in the 1980's began an uninterrupted trend toward low-noise, high speed, spatial and temperature resolution instruments that provide digital data in real time. The introduction of the PC and the subsequent orders of magnitude improvements in speed, bandwidth and processing power that followed have enabled the development of sophisticated processing algorithms on data from the IR camera in near-real time. Taken together, these advances have provided researchers with the means to dramatically extend the range of TNDT applications in terms of materials, resolution and precision.

Early efforts in TNDT were focused on flaw detection, limited primarily by camera performance, and usually confined to large aspect ratio voids or delamination in materials that were "IR-friendly" in terms of thermal diffusivity and emissivity (even then, samples were often painted black to optimize emissivity). As the aforementioned advances in camera and PC technologies became available, TNDT flaw detection performance improved and the limitations on materials and flaw size relaxed accordingly. The PC advances allowed researchers to develop and implement dedicated signal processing algorithms, operating on each individual pixel time history. While these algorithms were based on well-known mathematical operations (e.g. Fourier transform, polynomial fitting, matrix diagonalization), it is only in the past 10-15 years that they can be performed on the massive amount of data in an IR image sequence on a time scale that is realistic for commercial implementation. Three processing methods have emerged as the most widely used in modern practice: Thermographic Signal Reconstruction (TSR), Pulse Phase (PP) and Principal Component Analysis (PCA). While the underlying mathematics and format of the final result from each varies significantly, they operate on identical data sets, and are subject to the same fundamental physical limitations imposed by thermal diffusion. The have been compared and benchmarked in numerous studies, and what has emerged is that they all are capable of detecting flaws that are not detectable in the unprocessed image sequence.
\end{abstract}

Despite the evolution and maturation of TNDT, the method is still most widely used for flaw detection, and many of the assumptions, benchmarks and procedures that are in place today are based on the 


\subsection{1/qirt.2016.b}

traditional flaw-detection paradigm. This is unfortunate, since the needs of the industrial marketplace often require more nuanced inspection, e.g. in Quality Assurance (QA) where measurement of dimensional or thermophysical properties may be required, or where some aspect of the manufacture of a part (e.g. cure, mixture of chemical constituents, density) meets the necessary specification. In such application scenarios a discrete flaw may not be a likely, or even relevant, event, so rather than finding a flaw in a good part, the task is to compare "good" part to a "perfect" one, with the further complication that the inspection must be performed quickly, and preferably, automatically, without the intervention of a human inspector. In fact, TNDT is currently being applied to such tasks with increasing frequency, and in many cases, in completely automated systems. However, these systems are often application specific, and bear little resemblance to the early general purpose systems. In this talk, we will discuss the issues involved in implementing thermography in actual industrial applications, focusing on defining application requirements and identifying the most direct and robust hardware and processing solutions that meet those specific requirements. 